Article

\title{
Methods for Identification of CA125 from Ovarian Cancer Ascites by High Resolution Mass Spectrometry
}

\author{
Florian Weiland ${ }^{1, \dagger}$, Katarina Fritz ${ }^{1, \dagger}$, Martin K. Oehler ${ }^{2}$ and Peter Hoffmann ${ }^{1, *}$
}

1 Adelaide Proteomics Centre, School of Molecular and Biomedical Science, University of Adelaide; Adelaide 5005, Australia; E-Mails: florian.weiland@adelaide.edu.au (F.W.); katarina.fritz@medunigraz.at (K.F.)

2 Research Centre for Reproductive Health, Robinson Institute, University of Adelaide; Adelaide 5005, Australia; E-Mail: martin.oehler@adelaide.edu.au

$\dagger$ These authors contributed equally to this work.

* Author to whom correspondence should be addressed; E-Mail: peter.hoffmann@adelaide.edu.au; Tel.: +61-8-8313-5507; Fax: +61-8-8313-4362.

Received: 15 May 2012; in revised form: 11 July 2012 / Accepted: 24 July 2012 / Published: 9 August 2012

\begin{abstract}
CA125 is the most widely used tumour marker in ovarian cancer with unsatisfactory sensitivity and specificity especially at early stage. It is quantified by antibody-based immunoassays; however different molecular weight isoforms have been described in the literature which have never been validated by mass spectrometry, potentially affecting the diagnostic accuracy and clinical reliability of the test. In this study, CA125 was detected by Western blot and its identity confirmed by mass spectrometry. Two-dimensional (2D) gel electrophoresis in combination with mass spectrometry revealed that positive Western blot signals up to $500 \mathrm{kDa}$ are most likely false-positive interactions of M11-like and OC125-like antibodies. Fibronectin, identified as one of these false-positive interaction partners, increased the reading for CA125 in a first generation ELISA significantly $(p=0.02)$. The existence of low-molecular weight isoforms of CA125 is therefore questionable and is most likely reflecting cross-reactivity of the antibodies with other proteins. This would explain the conflicting reports on the molecular structure of CA125 and also the inconsistency of CA125 levels by different ELISAs. Our results are also the first steps towards a mass spectrometric assay for CA125 quantification, which would improve sensitivity and reliability.
\end{abstract}


Keywords: CA125; mucin 16; mass spectrometry; ovarian cancer; antibody; false-positives

\section{Introduction}

Cancer antigen 125 (CA125), also known as mucin-16 (MUC16), is the most widely used tumour marker in ovarian cancer, and considered the "gold standard". Serum level of CA125 is used to monitor response to chemotherapy, relapse, and disease progression in ovarian cancer patients. However, its role for screening and early detection of ovarian cancer is limited due to a low sensitivity and specificity. Nevertheless, it is used for diagnostic purposes in combination with other methods such as transvaginal ultrasonography [1].

CA125 was first detected by Bast et al. in 1981 using a murine monoclonal antibody purified following immunization of mice with a human ovarian cancer cell line [2]. This antibody reacted with the majority of ovarian carcinoma cells and appeared to be nonreactive with non-malignant tissues. The antibody was called OC125 and the corresponding class of antibodies that recognize the same epitope are known as OC125-like antibodies. To date, several different classes of antibodies recognizing CA125 are known, with OC125-like and M11-like antibodies the most frequently used. These antibodies provide the basis for CA125-detection by enzyme linked immunosorbent assay (ELISA). First generation ELISAs use the same antibody for catching and tracing of the antigen, which renders a repetition of the epitope necessary. In contrast, second generation assays use OC125-like antibodies as catchers and M11-like antibodies as tracers and vice versa. Davelaar et al. [3] tested both first and second generation assays, concluding that both provide results with concordant tendencies; however, for monitoring a single patient, assays should not be interchanged due to varying values within the different assays. The cut-off value established for CA125 levels is $35 \mathrm{kU} / \mathrm{L}$ [4]. However, various malignant and benign conditions such as pregnancy, cardiovascular and liver diseases give rise to elevated CA125 levels [5-7]. Due to this lack of specificity, CA125 levels are only considered for monitoring treatment response and follow-up of ovarian cancer patients.

Thus far, characterization of CA125 has relied almost exclusively on the usage of antibodies. CA125 forms with molecular masses ranging from $110 \mathrm{kDa}$ to more than $2000 \mathrm{kDa}$ have been reported [8-13]. The protein has commonly been identified in the interface of stacking and separating gel, which points to a very high molecular weight $[14,15]$. Identification of CA125 by mass spectrometry has been described very rarely in literature [16-18]. High accuracy MS and MS/MS data is only available for CA125 with a mass of 2-3 MDa [19], for lower molecular weight forms no such data is available.

Here we present high accuracy MS and MS/MS data for CA125 and our findings question the existence of molecular mass isoforms below $500 \mathrm{kDa}$. Furthermore, the reliability of CA125 detection by probing with antibodies is challenged as we identified several antigens interacting with the M11-like and OC125-like antibody resulting in false-positive signals. One of those antigens, fibronectin, elevated the CA125 reading significantly in a first generation M11-like ELISA. The reliability of first generation ELISA to determine CA125 levels is therefore doubtful. All together, the findings explain the conflicting reports on the molecular structure of CA125 (reviewed in [20]) and also the inconsistencies of CA125 levels measured by different ELISAs [3]. 


\section{Results}

\subsection{One-Dimensional (1D) Gel Electrophoresis of Human Ascites}

As the various molecular mass isoforms of CA125 span over a range of 110-2000 kDa [8-13], we used a $T=3-8 \%$ polyacrylamide gel to resolve all forms simultaneously. A total of $94 \mu \mathrm{g}$ protein from patient (P) 517 ascites was loaded onto a 1D gel. The Western blots showed positive signals from the interaction of the M11-like antibody at molecular masses of approximately $117 \mathrm{kDa}, 200 \mathrm{kDa}$, $250 \mathrm{kDa}, 460 \mathrm{kDa}, 500 \mathrm{kDa}$ and a positive signal at the edge of the sample well (Figure S1). These protein bands were excised from a Coomassie stained gel (Figure S1) and subjected to identification by mass spectrometry (Table S1). With this approach we were not able to identify CA125 by massspectrometry in any excised bands.

\subsection{Enrichment of CA125 by Size Exclusion Chromatography}

In order to identify CA125 by mass spectrometry, we applied size exclusion chromatography (SEC) (7) to reduce sample complexity and to enrich CA125. Ascites of P86 was chosen for SEC as it has a higher level of CA125 in comparison to P517. $500 \mu \mathrm{L}$ of ascites was separated by SEC and the derived fractions were tested by dot-blotting for reactivity with the M11-like antibody (Figure S2). Fraction 14 and 15 showed the highest intensity signal for CA125. Then $200 \mu \mathrm{L}$ of each fraction was precipitated with a ReadyPrep 2D clean-up kit. Proteins from each fraction were separated on a 1D gel and electro-blotted (Figure 1A). The bands showing positive signals were excised from a Coomassie stained 1D gel (Figure 1B) and identified by mass spectrometry (Table S2). Analysis by tandem mass spectrometry of band IV on the very top of the 1D gel identified twenty-one unique peptide sequences of CA 125; five of them exhibited MASCOT scores over the significance threshold (Expectation $\leq 0.05$, see Tables 1 and S2). However, CA125 could not be identified in the excised bands I, II, III and V.

Figure 1. (A) Western blot of SDS-PAGE ( $T=3-8 \%)$ of size exclusion chromatography (SEC) fractions 14 and 15, M indicates the molecular mass marker lane; (B) Coomassie brilliant blue stained SDS PAGE $(T=3-8 \%)$ gel of SEC fractions 14 and 15. M indicates the molecular mass marker lane, roman numbers indicate bands corresponding to positive signals in western blot, which were subsequently identified by mass-spectrometry, CA125 was identified in Band IV by mass spectrometry (see Tables 1 and S1).

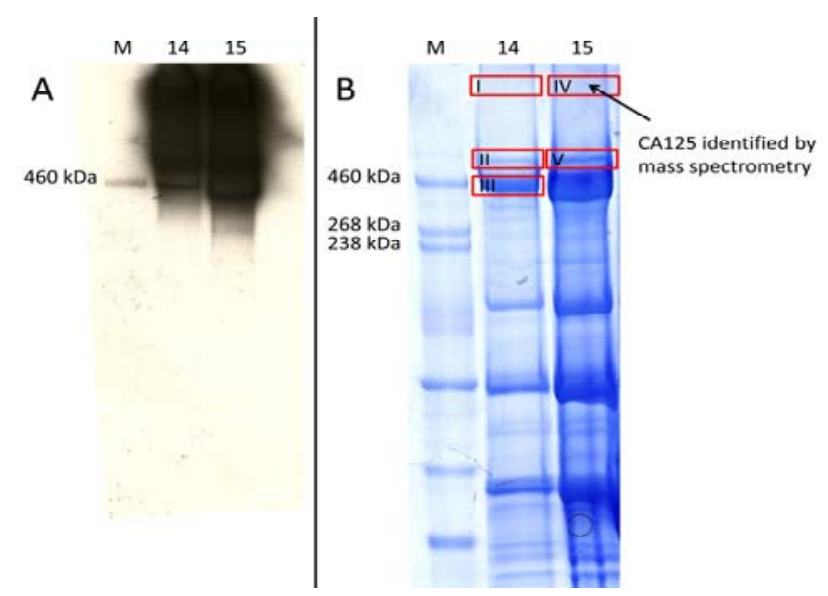


Table 1. MASCOT search results for peptides of CA125 from SDS-PAGE gel band IV (Figure 1B) identified by a HCT Ultra 3D Ion Trap mass spectrometer. The score of the peptides written in bold exceed the significance threshold (Expectation $\leq 0.05$ ).

\begin{tabular}{ll}
\hline Protein name: & Mucin-16 \\
Mass [Da]: & 2359682 \\
Score: & 577 \\
Matches: & $33(5)$ \\
Sequences: & $21(5)$ \\
Sequence Coverage: UniProt Acc.: & $1 \%$ MUC16_HUMAN \\
\hline
\end{tabular}

\begin{tabular}{|c|c|c|c|c|c|c|}
\hline Observed $^{a}$ & $\mathrm{z}^{b}$ & $\operatorname{Mr}\left(\right.$ calc) ${ }^{c}$ & Delta $^{d}$ & Score $^{e}$ & Expect $^{f}$ & Peptide \\
\hline 654.42 & 2 & 1306.6418 & 0.1836 & 84 & $6.80 \mathrm{E}-07$ & R.NSLYVNGFTHR.S \\
\hline 722.45 & 2 & 1442.6572 & 0.2282 & 68 & 2.70E-05 & K.DGAATGVDAICTHR.L \\
\hline 875.05 & 2 & 1747.8628 & 0.2226 & 52 & 0.001 & K.LTNDIEELGPYTLDR.N \\
\hline 655.92 & 2 & 1309.6085 & 0.2170 & 44 & 0.0069 & K.NTSVGPLYSGCR.L \\
\hline 779.93 & 2 & 1557.7206 & 0.1249 & 41 & 0.014 & K.QEAATGVDTICTHR.V \\
\hline 654.90 & 2 & 1307.6259 & 0.1596 & 35 & 0.06 & R.DSLYVNGFTHR.S \\
\hline 625.91 & 2 & 1249.6204 & 0.1851 & 34 & 0.076 & R.GSLYVNGFTHR.T \\
\hline 485.32 & 2 & 968.6018 & 0.0236 & 32 & 0.11 & R.LTLLRPEK.D \\
\hline 485.32 & 2 & 968.6018 & 0.0236 & 32 & 0.12 & R.LTLLRPEK.D \\
\hline 485.35 & 2 & 968.6018 & 0.0836 & 31 & 0.12 & R.LTLLRPEK.D \\
\hline 555.37 & 2 & 1108.5877 & 0.1378 & 31 & 0.14 & R.LDPLNPGLDR.E \\
\hline 875.00 & 2 & 1747.8628 & 0.1226 & 29 & 0.22 & K.LTNDIEELGPYTLDR.N \\
\hline 532.38 & 2 & 1062.5346 & 0.2109 & 28 & 0.32 & K.ELGPYTLDR.N \\
\hline 532.38 & 2 & 1062.5346 & 0.2109 & 28 & 0.34 & K.ELGPYTLDR.N \\
\hline 642.40 & 2 & 1282.5976 & 0.1879 & 26 & 0.46 & K.STSVGPLYSGCR.L \\
\hline 598.41 & 2 & 1194.5921 & 0.2134 & 25 & 0.49 & R.EQLYWELSK.L \\
\hline 596.67 & 3 & 1786.8268 & 0.1614 & 25 & 0.51 & R.SEKDGAATGVDAICTHR.L \\
\hline 642.40 & 2 & 1282.5976 & 0.1879 & 25 & 0.57 & K.STSVGPLYSGCR.L \\
\hline 485.39 & 2 & 968.6018 & 0.1636 & 22 & 0.7 & R.LTLLRPEK.D \\
\hline 399.85 & 2 & 797.5123 & 0.1731 & 22 & 0.73 & R.VLQGLLR.S \\
\hline 654.90 & 2 & 1307.6259 & 0.1596 & 24 & 0.81 & R.DSLYVNGFTHR.S \\
\hline 679.95 & 2 & 1357.7605 & 0.1249 & 23 & 1 & R.GIIELGPYLLDR.G \\
\hline 570.42 & 2 & 1138.6022 & 0.2232 & 20 & 1.3 & R.VAIYEEFLR.M \\
\hline 662.46 & 2 & 1322.7082 & 0.1973 & 20 & 1.3 & R.DIQDKVTTLYK.G \\
\hline 532.33 & 2 & 1062.5346 & 0.1109 & 22 & 1.4 & K.ELGPYTLDR.N \\
\hline 598.41 & 2 & 1194.5921 & 0.2134 & 21 & 1.4 & R.EQLYWELSK.L \\
\hline 436.95 & 3 & 1307.6259 & 0.2023 & 20 & 1.8 & R.DSLYVNGFTHR.S \\
\hline 875.00 & 2 & 1747.8628 & 0.1226 & 20 & 1.9 & K.LTNDIEELGPYTLDR.N \\
\hline 594.27 & 3 & 1779.9016 & -0.1135 & 15 & 5.5 & K.QVFHELSQQTHGITR.L \\
\hline 663.40 & 2 & 1324.6735 & 0.1119 & 11 & 13 & R.LDPTGPGLDRER.L \\
\hline 628.43 & 2 & 1254.8064 & 0.0391 & 10 & 18 & R.VLQGLLKPLFK.S \\
\hline
\end{tabular}

${ }^{a}$ Observed mass/charge of the peptide in Da; ${ }^{b}$ Charge state of the peptide ${ }^{c}$ Theoretical peptide mass in Da;

${ }^{d}$ difference between the observed and the theoretical mass in Da; ${ }^{e}$ MASCOT score; ${ }^{f}$ Expectation of a random match, derived from the MASCOT score; ${ }^{g}$ Mass accuracy in parts per million. 


\subsection{Two-Dimensional (2D) Electrophoresis of Human Ascites}

Two-Dimensional gel electrophoresis (2DE) facilitates the separation of proteins by their isoelectric points (pI) and their molecular weights, thus we hypothesized that this might separate CA125 isoforms from other proteins with equivalent molecular weight. As 2DE offers a higher protein loading capacity than 1D SDS-PAGE, we used $225 \mu \mathrm{g}$ of protein isolated from P517 ascites without prior CA125 enrichment by SEC. The sample was labeled with Cy2 and separated by $\mathrm{pI}$ in the range of $\mathrm{pH} 3-10$. Second dimension was performed in a $T=4-12 \%$ SDS-PAGE gel. The proteins from the $2 \mathrm{D}$ gel were electroblotted onto a low-fluorescent PVDF membrane, scanned with an Ettan DIGE Imager and afterwards proteins interacting with the M11-like and OC125-like antibodies were detected by ECL (Figure 2(A,B)). By overlaying the two images we were able to locate the proteins which gave a positive signal accurately. Corresponding proteins were excised from a Coomassie stained 2D gel (225 $\mu \mathrm{g}$ protein load, Figure 2(C,D)) and identified by mass spectrometry (Tables S3 and S4). The results of the identification showed that CA125 could not be identified from these $2 \mathrm{D}$ gels.

Figure 2. (A and B): 2D Western blot (225 $\mu \mathrm{g}$ protein load, P517 ascites, IPG 3-10NL, $T=4-12 \%$ ), probed with HRP conjugated M11-like antibody (A) or OC125-like antibody and secondary HRP-conjugated antibody (B). White circles in (B) correspond to signals exclusive in their interaction with OC125-like antibody. (C and D): Coomassie stained 2D gel (225 $\mu$ g protein load, P517 ascites, IPG 3-10NL, $T=4-12 \%)$, numbers indicate protein spots subjected to identification by mass spectrometry (see Tables S3 and S4 respectively); these spots co-locate with the signals from the western blots shown in Figure 2A (C) or Figure 2B (D) respectively. The star in Figure 2B indicates proteins already indentified in Figure 2C, corresponding to protein spots 9-12. The red circle in Figure 2A indicates the signal obtained by a M11-like antibody (subsequently identified as fibronectin (see Table S3); the red circle in Figure 2B indicates the missing signal for fibronectin when probing with an OC125-like antibody.
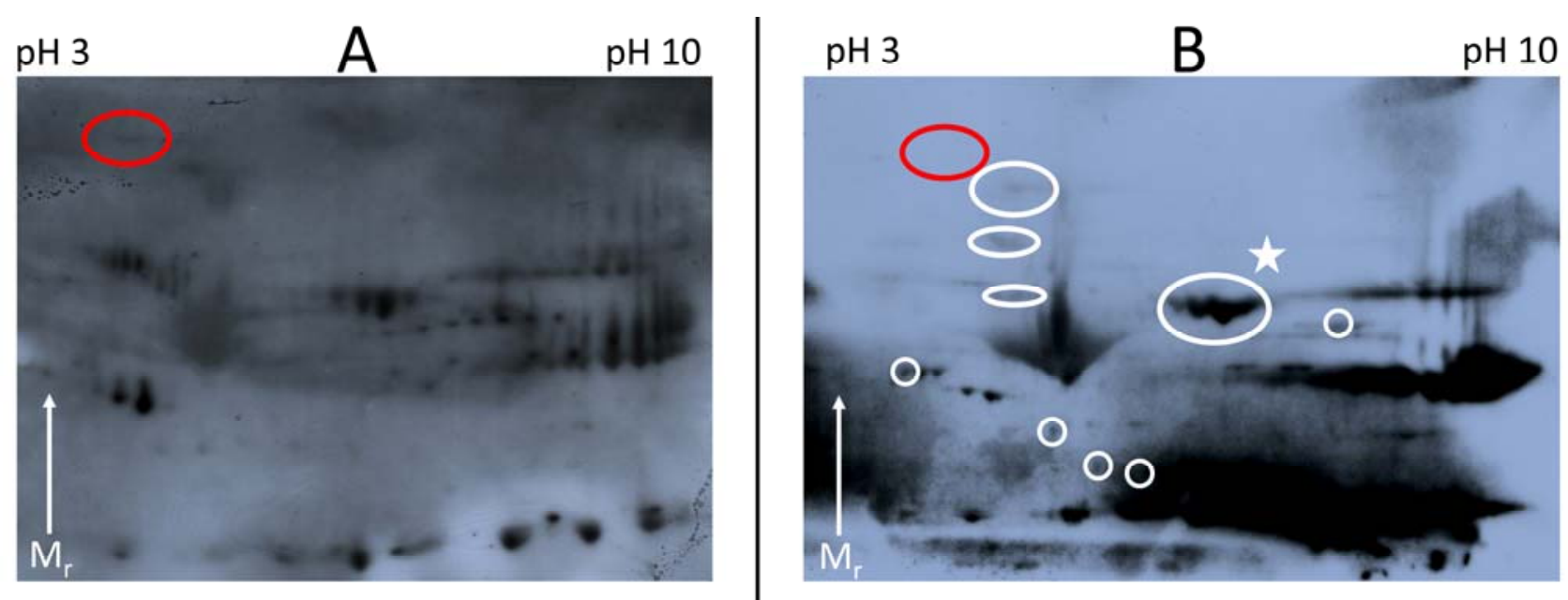
Figure 2. Cont.
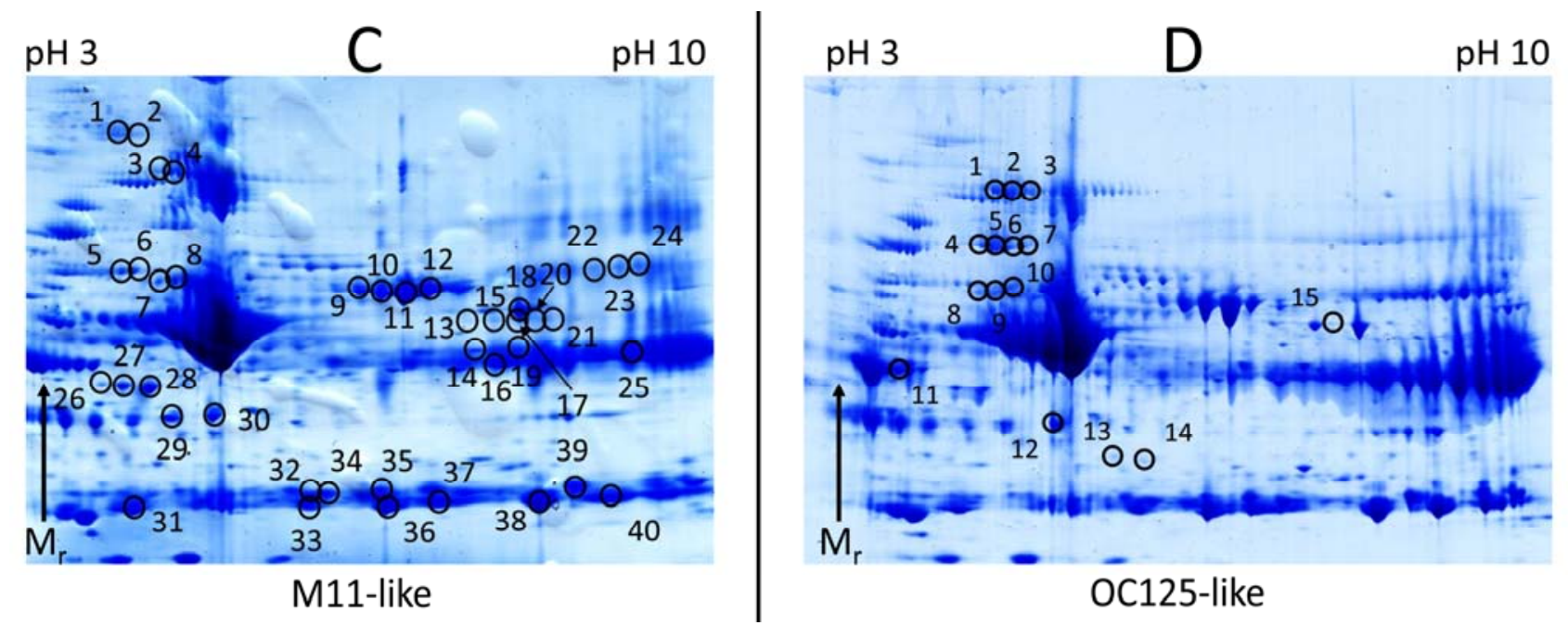

\subsection{Elisa of Human Serum Spiked with Fibronectin}

To verify that these false-positive signals on the $2 \mathrm{D}$ western blot were not derived from CA125 co-localization, but were resulting from an interaction between the M11-like antibody and those protein identified by mass spectrometry, fibronectin was chosen for spiking experiments. This protein was selected as it was identified in 1D gel bands and 2D gel protein spots by MS (1D: Figure S1, Table S1 (Band III and IV), 2D: Figure 2(A,C) (Spot number 1 and 2, Table S3) and was commercially available. We spiked $80 \mu \mathrm{g}$ of fibronectin into $100 \mu \mathrm{L}$ of 1:20 diluted P95 serum (as this M11-like-only ELISA Kit, according to the manufacturer, is only suitable for serum) and performed an ELISA for CA125. Accordingly, the spiked fibronectin represented $\sim 2.3 \%$ of the total protein amount, based on a serum concentration of $7000 \mathrm{mg} / \mathrm{dL}$. Normal serum levels of fibronectin are approximately $200 \mu \mathrm{g} / \mathrm{mL}$ [21], meaning the spiked fibronectin levels were in a 80-fold excess (on a 1:20 dilution of serum basis, this equals approximately a 4-fold excess in undiluted serum). We chose this unrealistically high excess as we were assuming that normal fibronectin amounts do not increase the CA125 reading significantly, given that serum from healthy people has a CA125 level below $35 \mathrm{kU} / \mathrm{L}$. A Mann-Whitney U-test showed that the spiked serum has a statistical significantly $(p=0.02)$ (see Figure 3C) increased reading for CA125 in a M11-like-only sandwich ELISA by an average of $980 \mathrm{kU} / \mathrm{L}$ (compared to the unspiked P95 serum). This means that a 1-fold excess of fibronectin raises the CA125 reading by around $12 \mathrm{kU} / \mathrm{L}$, thus normal serum levels of fibronectin are below the critical reading of $35 \mathrm{kU} / \mathrm{L}$. To test the influence of fibronectin on a clinically used M11-like and OC125-like sandwich ELISA, we repeated above mentioned experiment with a clinically approved sandwich ELISA, but no increased CA125 reading was detected (data not shown). 
Figure 3. (A) Measured values in absorbance units for CA125 standards (0-400 kU/L) (blue), 1:20 diluted P95 serum (green) and 1:20 diluted P95 serum spiked with $80 \mu \mathrm{g}$ fibronectin (red). The values obtained from P95 serum and spiked P95 serum were used for the Mann-Whitney U test; (B) Standard curve obtained by plotting the detected absorbance units against the $\mathrm{kU} / \mathrm{L}$ of the CA125 standards. This curve was used to calculate the CA125 concentration of the used samples (C) CA125 concentration measured by a first generation ELISA (M11-like). Green: P95 serum, red: P95 serum spiked with $80 \mu \mathrm{g}$ fibronectin. The increase in reading is statistically significant $(* p<0.05)$. Error bars indicate standard deviation of measured values within the respective sample; (D) CA125 concentrations measured by a clinically used second generation ELISA. I: $500 \mu \mathrm{L}$ P607 serum $+100 \mu \mathrm{L}$ $\mathrm{H}_{2} \mathrm{O}$; II: $500 \mu \mathrm{L}$ P607 serum $+100 \mu \mathrm{L}$ apo-serotransferrin $(100 \mathrm{mg} / \mathrm{mL})$; III: $500 \mu \mathrm{L}$ P607 serum $+100 \mu \mathrm{L}$ holo-serotransferrin $(100 \mathrm{mg} / \mathrm{mL})$. The increase in the reading for CA125 is statistically significant (*) between apo-serotransferrin (II) and unspiked serum (I) and statistically highly significant $(* * p<0.01)$ between holo-serotransferrin (III) and unspiked serum (I). Error bars indicate the standard deviation of the measured values in the respective sample.

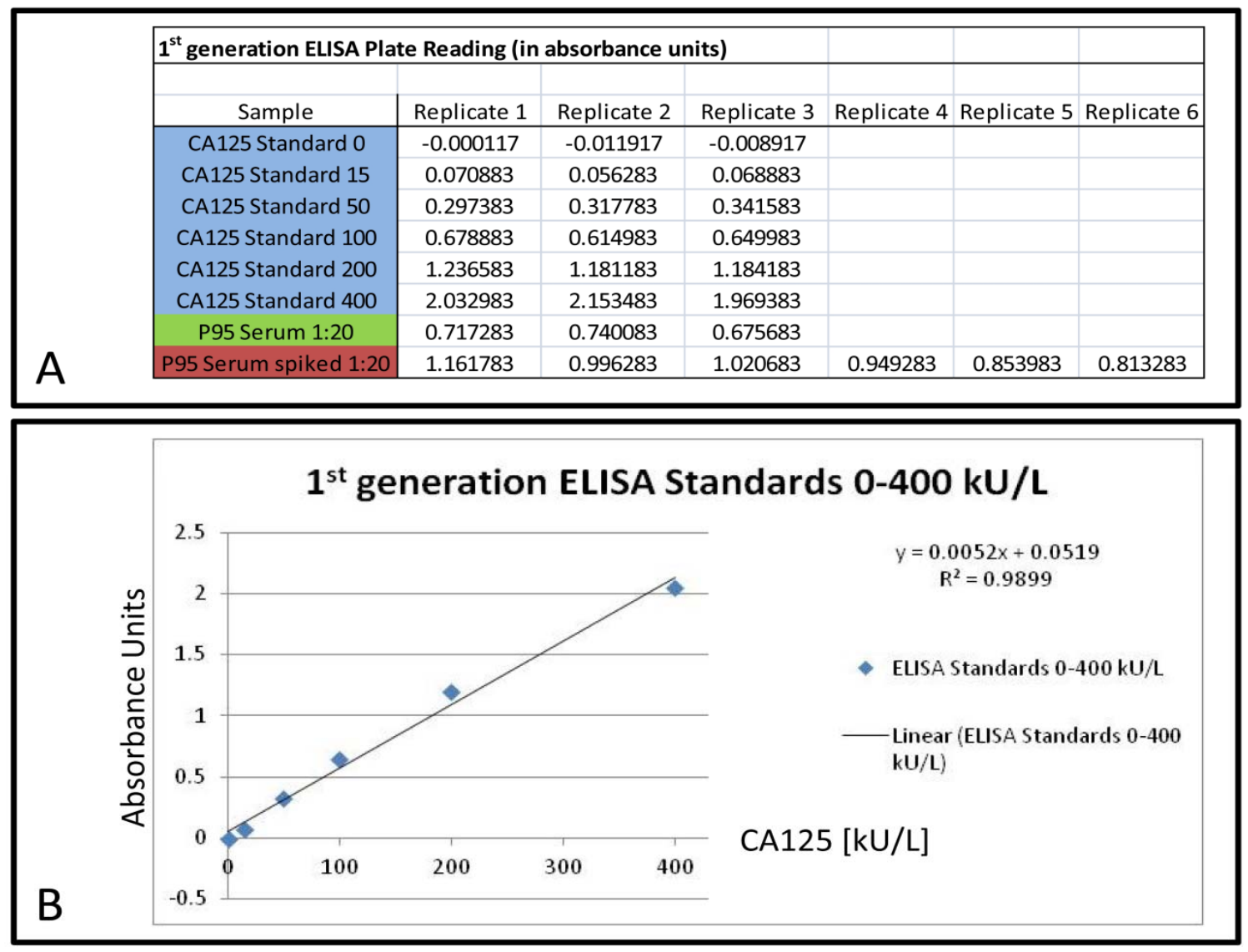


Figure 3. Cont.
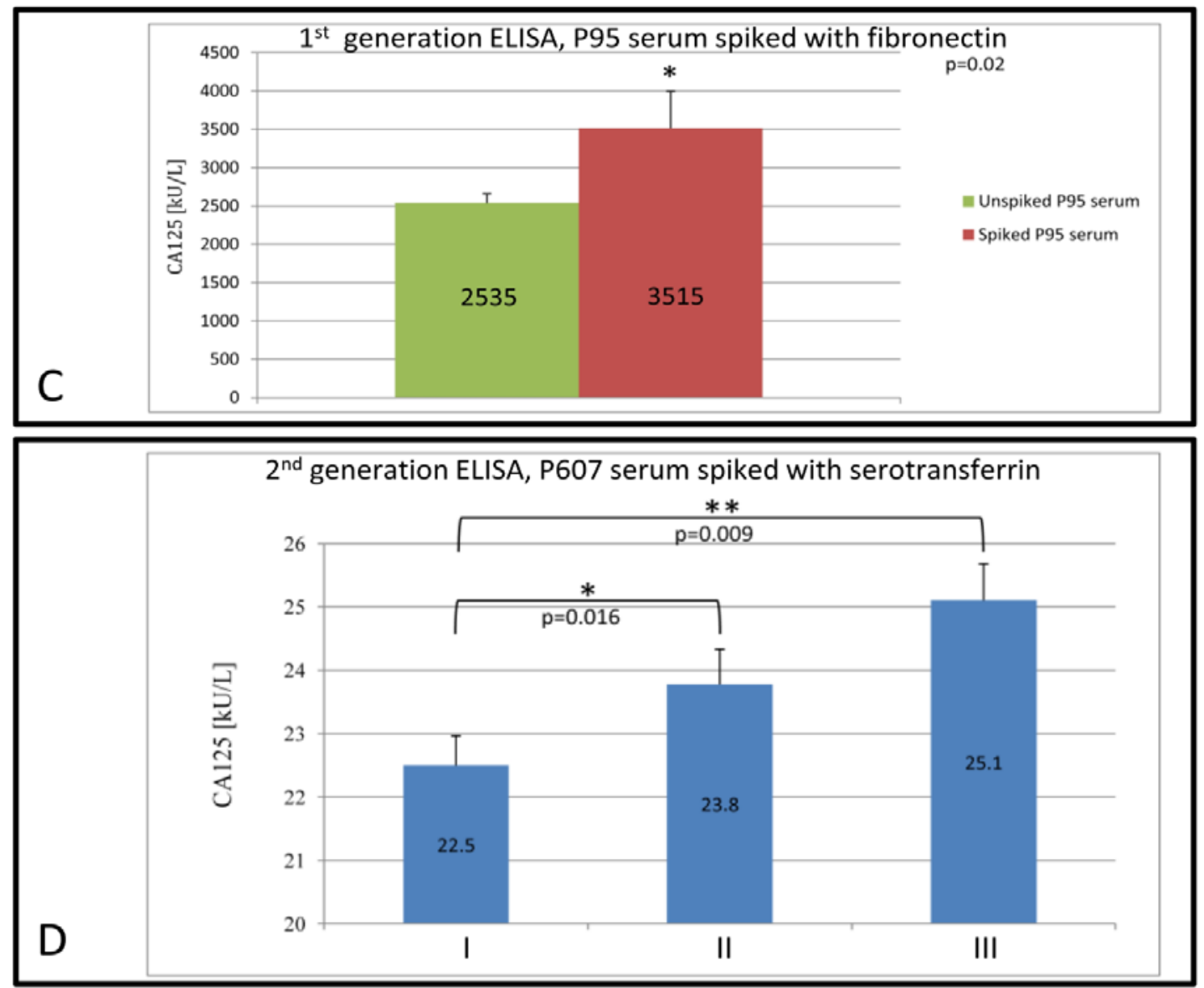

\subsection{Elisa of Human Serum Spiked with Serotransferrin}

Serotransferrin was detected as a protein giving a false-positive signal with both M11-like and OC125-like antibodies (see Figure 2). Therefore it was hypothesized that it would increase the CA125 reading in a clinically used, second generation ELISA. Based on a normal serotransferrin serum concentration of $2.04-3.60 \mathrm{mg} / \mathrm{mL}$ [22], $500 \mu \mathrm{L}$ of $\mathrm{P} 607$ serum was spiked with $10 \mathrm{mg}$ apo-serotransferrin and holo-serotransferrin respectively, which equals an approximate 5-fold increase of this protein. The samples were given to the Institute of Medical and Veterinary Sciences, Adelaide (IMVS) to measure the CA125 concentration. Both samples with spiked serotransferin showed a statistically significant increase in CA125 reading (apo-serotransferrin: $p=0.016$; holo-serotransferrin: $p=0.009$ ); however, the increase was only minor. The unspiked P607 serum had a CA125 reading of $22.5 \mathrm{kU} / \mathrm{L}$, the P607 serum spiked with apo- and holo-serotransferrin showed a CA125 reading of 23.8 and $25.1 \mathrm{kU} / \mathrm{L}$, respectively (see Figure 3D).

\subsection{High Accuracy Mass Spectrometry of CA125}

Mass spectrometric data for CA125 has rarely been reported and MS/MS data is nearly non-existent in the literature. To obtain high accuracy mass spectrometric data we performed a filter aided digest of the SEC fractions 13-17, as they gave positive signals when probed with an M11-like antibody (see Figure S2). Before analyzing these samples on a LTQ Orbitrap XL mass spectrometer, all digested 
fractions were tested for LC-MS incompatible polymer contamination with MALDI mass spectrometry and subsequently fraction 14 had to be excluded from high accuracy MS (data not shown). We chose the Orbitrap for this task as this mass spectrometer can achieve a mass accuracy $<5$ ppm with a $95 \%$ probability [23]. CA125 could be identified in fractions 15-17 (see Table 2). The combined data of those fractions displays 32 unique peptides of CA125; while 16 of them have a MASCOT score which is above the significance threshold (Expectation $\leq 0.05$ ), the mass accuracy is in the range of 0.03 to $2.25 \mathrm{ppm}$ (see Table 2, MS/MS spectra of statistically significant peptides in Figure S3). The other 16 are despite their lower score likely CA125 peptides because they were measured with high accuracy between 0.15 and $3.53 \mathrm{ppm}$, which is a similar accuracy to the 16 peptides above the threshold.

Table 2. Combined MASCOT search results for SEC fractions 15-17 in which CA125 was identified by a LTQ Orbitrap XL mass spectrometer. The score of the peptides written in bold exceed the significance threshold (Expectation $\leq 0.05$ ).

\begin{tabular}{|c|c|c|c|c|c|c|}
\hline Observed $^{a}$ & $\mathbf{z}^{b}$ & $\operatorname{Mr}\left(\right.$ calc) ${ }^{c}$ & ppm ${ }^{d}$ & Score $^{e}$ & Expect $^{f}$ & Peptide \\
\hline 748.8278 & 2 & 1495.64 & 0.60 & 52 & $4.60 \mathrm{E}-05$ & K.SYFSDCQVSTFR.S \\
\hline 798.7573 & 3 & 2393.249 & 0.60 & 56 & $5.90 E-05$ & R.LTLLRPEKDGAATGVDAICTHR.L \\
\hline 570.3081 & 2 & 1138.602 & -0.50 & 44 & 0.0008 & R.VAIYEEFLR.M \\
\hline 399.7638 & 2 & 797.5123 & 1.05 & 37 & 0.00099 & R.VLQGLLR.S \\
\hline 655.812 & 2 & 1309.609 & 0.75 & 42 & 0.0011 & K.NTSVGPLYSGCR.L \\
\hline 721.3632 & 2 & 1440.714 & -1.80 & 39 & 0.003 & K.HGAATGVDAICTLR.L \\
\hline 642.3064 & 2 & 1282.598 & 0.51 & 36 & 0.0039 & K.STSVGPLYSGCR.L \\
\hline 596.814 & 4 & 2383.228 & -0.32 & 38 & 0.0047 & R.LTLLRSEKDGAATGVDAICTHR.L \\
\hline 654.3267 & 2 & 1306.642 & -2.25 & 37 & 0.0058 & R.NSLYVNGFTHR.S \\
\hline 601.8525 & 2 & 1201.689 & 1.02 & 34 & 0.0078 & R.VLQGLLGPMFK.N \\
\hline 523.6422 & 3 & 1567.905 & 0.03 & 30 & 0.011 & R.LTLLRPEKDGVATR.V \\
\hline 607.8801 & 2 & 1213.743 & 1.87 & 27 & 0.014 & R.VLQGLLSPIFK.N \\
\hline 837.1103 & 3 & 2508.312 & -1.12 & 30 & 0.022 & R.LTLLRPEKQEAATGVDTICTHR.V \\
\hline 677.8256 & 2 & 1353.635 & 1.48 & 28 & $\mathbf{0 . 0 3 7}$ & K.NTSIGPLYSSCR.L \\
\hline 798.1088 & 3 & 2391.306 & -0.49 & 25 & 0.04 & R.LTLLRPEKHGAATGVDAICTLR.L \\
\hline 594.3086 & 3 & 1779.902 & 1.29 & 28 & 0.049 & K.QVFHELSQQTHGITR.L \\
\hline 711.1059 & 4 & 2840.395 & -0.15 & 27 & 0.053 & R.LTSLRPEKDGAATGMDAVCLYHPNPK.R \\
\hline 679.8878 & 2 & 1357.761 & 0.41 & 25 & 0.077 & R.GIIELGPYLLDR.G \\
\hline 628.4118 & 2 & 1254.806 & 2.14 & 12 & 0.086 & R.VLQGLLKPLFK.S \\
\hline 1007.534 & 2 & 2013.053 & 0.59 & 26 & 0.088 & K.LSQLTHGITELGPYTLDR.H \\
\hline 947.8076 & 3 & 2840.395 & 2.15 & 25 & 0.089 & R.LTSLRPEKDGAATGMDAVCLYHPNPK.R \\
\hline 514.2987 & 3 & 1539.873 & 0.68 & 16 & 0.43 & R.LTLLRPEKDGAATR.V \\
\hline 717.8562 & 2 & 1433.697 & 0.39 & 17 & 0.57 & K.VDAICTYRPDPK.S \\
\hline 598.3046 & 2 & 1194.592 & 2.12 & 15 & 0.61 & R.EQLYWELSK.L \\
\hline 607.3171 & 3 & 1818.926 & 1.65 & 17 & 0.69 & K.SPGLNREQLYWELSK.L \\
\hline 555.9742 & 3 & 1664.9 & 0.54 & 13 & 1.2 & R.RVDRVAIYEEFLR.M \\
\hline 514.2987 & 3 & 1539.873 & 0.68 & 10 & 1.8 & R.LTLLRPEKDGAATR.V \\
\hline 477.5088 & 4 & 1906.01 & -1.85 & 6 & 6.3 & K.NTSVGPLYSGCRLTLLR.S \\
\hline 862.958 & 2 & 1723.903 & -1.02 & 5 & 7.8 & K.DPEILSWTIPPSIEK.T \\
\hline
\end{tabular}


Table 2. Cont.

\begin{tabular}{|c|c|c|c|c|c|c|}
\hline Observed $^{a}$ & $\mathrm{z}^{b}$ & $\operatorname{Mr}\left(\right.$ calc) ${ }^{c}$ & $\mathbf{p p m}^{d}$ & Score $^{e}$ & Expect $^{f}$ & Peptide \\
\hline 647.5736 & 4 & 2586.261 & 1.71 & 5 & 8.8 & K.HEAATGVDTICTHRVDPIGPGLDR.E \\
\hline 702.6927 & 3 & 2105.058 & -0.61 & 4 & 11 & K.KDGAATKVDAICTYRPDPK.S \\
\hline 848.0892 & 3 & 2541.237 & 3.53 & 1 & 19 & K.SKLSLTPGLMETSISEETSSATEK.S \\
\hline
\end{tabular}

\section{Discussion}

The current standard procedure for the detection of CA125 in biofluids is SEC in combination with 1D-SDS-PAGE, western-blotting and probing with M11-like and/or OC125 type of antibodies [18,24]. Reports on the verification of CA125 signals by mass spectrometry are rare and lack detail [16-19]. This is especially unsatisfactory for CA125, as it is a very large protein and this raises the probability for random peptide matches, particularly as no statistical scoring is given. Here we report the mass spectrometric identification of CA125 as a high molecular weight protein from a $1 \mathrm{D}$ gel after enrichment by denaturing SEC (see Figure 1 and Table 1). Additionally, the positive SEC fractions have been digested by trypsin and analyzed by an LTQ Orbitrap XL to obtain high accuracy mass spectrometric and MS/MS data of CA125. We identified 16 unique peptides of CA125; another 16 peptides were identified, but have MASCOT scores below the significance threshold (see Table 2). However, as we enriched for CA125 it is likely that these peptides are real discoveries and not random matches because of the high mass accuracy of the measurement (between 0.15 and $3.53 \mathrm{ppm}$ ). The mass accuracy of the identified significant peptides was equal or better than $2.25 \mathrm{ppm}$ (see Table 2); the false discovery rate on the peptide level in the fractions 15,16 and 17 was $1.65 \%, 1.85 \%$ and $2.07 \%$ respectively. These findings make it evident that it is possible to acquire sufficient MS data to verify signals from probing with anti-CA125 antibodies, instead of relying on immuno-blots alone. This is especially important as we show that there are cross-reactions with the M11-like antibody in at least one case. The positive signals observed on the 2D Western-blots (see Figure 2, Table S3 and S4) are caused by mostly the same proteins we also identified in a 1D gel (see Figure S1 and Table S1). Potentially, the low molecular weight forms of CA125 could be masked by co-migrating proteins in $1 \mathrm{D}$ and 2D gels or otherwise impossible to detect by mass spectrometry. However, we show that spiking of human serum with fibronectin increases the reading for CA125 significantly $(p=0.02)$ in a first generation M11-like ELISA (see Figure 3C). This confirms the existing of at least one false-positive interaction partner of M11-like antibodies. Spiking $80 \mu \mathrm{g}$ of fibronectin into 1:20 diluted serum resulted in an increase in the CA125 concentration measured by a first generation ELISA by $980 \mathrm{kU} / \mathrm{L}$. Therefore, the amount of spiked fibronectin raises the reading for CA125 in undiluted serum by only one twentieth of the measured increase, i.e., $49 \mathrm{kU} / \mathrm{L}$ (increase in reading divided by dilution factor). This would raise CA125 readings, in a first generation ELISA, over the clinically used cut-off value of $35 \mathrm{kU} / \mathrm{L}$. Importantly, as $80 \mu \mathrm{g}$ of fibronectin equals to an 4-fold excess in undiluted serum, a normal (1-fold) serum level of fibronectin, would contribute around $12 \mathrm{kU} / \mathrm{L}$ to the CA125 level, according to our experiment. This is below the established cut-off of $35 \mathrm{kU} / \mathrm{L}$. 
These findings raise reasonable doubt about the molecular properties of published low-molecular weight isoforms of CA125. Based on our results, it cannot be excluded that proteins cross-reacting with the CA125 antibodies have been characterized along the primary protein of interest. This may have lead to the mutually exclusive characteristics of CA125 in terms of carbohydrate content and predominant type of glycosylation $[8,20,25]$. Therefore, mass spectrometry is an indispensible tool for assessing the purity of CA125 preparations. The observed secondary reactions of the applied antibodies also raise questions about the low molecular weight isoforms of CA125. These forms have been identified so far exclusively by antibody probing and therefore independent evidence of CA125 identity is required.

Furthermore, we show that OC125-like and M11-like antibodies cross-react with different protein subsets (see Figure 2(A,B)). This provides one explanation for the varying levels of CA125 detected by different ELISAs [3] as distinct antibodies against CA125 are utilized. The reaction of the M11-like and OC125-like antibodies to different protein subsets gives also an explanation to the failure of fibronectin to raise the CA125 readings in a second generation ELISA, which employs M11-like and OC125-like antibodies. Fibronectin is only an interaction partner with M11-like antibodies, as spots 1 and 2 in Figure 2C (identified as fibronectin, see Tables S3 and S4) have no corresponding signal in Figure 2B. Serotransferrin was identified as a secondary interaction partner for M11-like and OC125-like antibodies and was able to raise the reading for CA125 in a second generation ELISA statistically significantly, however only minor slightly (Figure 3D). The reason for this is unknown, however it can be speculated that the cross-reaction of the different antibody types in the respective anti-CA125 antibody families is varying and the here used M11-like and OC125-like antibodies do not match the antibody strains applied by the IMVS. Therefore, this result should have only little impact on the clinical detection measurement of CA125. However, it can be argued that an increase in serum concentration of several proteins, affecting readings in the CA125 assay, could be cumulative and have a more significant impact on the clinical detection.

\section{Experimental Section}

This study was approved by the Human Ethics Committee of Adelaide University.

\subsection{Sample Preparation}

Ascites of two ovarian cancer patients (P517 and P86) was collected and submitted to the IMVS to determine the amount of CA125. The remaining ascites was aliquoted and stored at $-80{ }^{\circ} \mathrm{C}$. The CA125 concentrations of P517 and P86 ascites were $6609 \mathrm{kU} / \mathrm{L}$ and 36,000 kU/L, respectively.

Blood of ovarian cancer patients P95 and P607 was collected into clotting tubes (Greiner Bio-one), centrifuged at $3000 \mathrm{rpm}$ for $10 \mathrm{~min}$ at room-temperature and the plasma was removed and stored in $500 \mu \mathrm{L}$ aliquots at $-80^{\circ} \mathrm{C}$.

\subsection{D Electrophoresis}

Proteins from P517 ascites were precipitated using the ReadyPrep 2D clean up kit (Bio-Rad Laboratories) according to manufacturer's protocol and resuspended in TUC4\% buffer (7 M urea, 
$2 \mathrm{M}$ thiourea, 4\% (3-[(3-Cholamidopropyl)-dimethylammonio]-1-propansulfonat (CHAPS), 2\% Dithiothreitol (DTT), 2\% Pharmalyte 3-10 (GE Healthcare, Buckinghamshire, UK, $30 \mathrm{mM}$ Tris). The protein concentration was estimated using EZQ protein quantitation kit (Life technologies Corporation, Carlsbad, CA, USA). Prior to isoelectric focusing, $225 \mu \mathrm{g}$ of protein was labeled with Cy2 (GE Healthcare) according to manufacturer's protocol, deviating in the use of 200 pmol of CyDye label for $225 \mu \mathrm{g}$ of proteins, the labeling was omitted when the proteins were stained with Coomassie brilliant blue after 2D electrophoresis. IPG strips were rehydrated overnight in TUX $1 \%$ buffer (6 M Urea, 2 M Thiourea, 50\% Acetonitrile, 1\% CHAPS, 0.5 Pharmalyte 3-10 (GE Healthcare) and $200 \mathrm{mM}$ 2,2-Dithiodiethanol). Isoelectric focusing was performed on an IPGphor II (GE Healthcare) using anodic cup loading according to published protocols [26]. SDS-PAGE was carried out on Criterion XT gels (Bio-Rad Laboratories, Hercules, CA, USA) with a $T$-value (T) of $4-12 \%$ according to manufacturer's protocol. Proteins were stained with Coomassie brilliant blue overnight.

\subsection{Western Blot}

After SDS-PAGE, proteins were electroblotted onto a low-fluorescent PVDF membrane (Merck KGaA, Darmstadt, Germany) using a Criterion wet blotter (Bio-Rad). The membrane was then scanned on an Ettan DIGE imager (GE Healthcare) using the Cy2 channel. Two different anti-CA125 antibodies were used. In the first work-flow, the membrane was incubated with directly horse-radish peroxidase (HRP)-conjugated monoclonal anti-CA125 antibody (M11-like) (HyTest, Joukahaisenkatu, Finland). In the second work-flow the membrane was incubated with a mouse derived monoclonal OC125-like antibody (Thermo Fisher Scientific, Waltham, MA, USA), afterwards the membrane was incubated with an anti-mouse HRP-conjugated IgG (Rockland Immunochemicals, Gilbertsville, PA, USA). Both work-flows applied enhanced chemiluminescence (ECL) detection (Sigma-Aldrich, St. Louis, MO, USA).

\subsection{Size Exclusion Chromatography}

Prior to gel filtration, $1 \mathrm{~mL}$ of ascites was thawed and centrifuged at $2500 \mathrm{~g}$ and $4{ }^{\circ} \mathrm{C}$ for $10 \mathrm{~min}$ to remove cells. Supernatant was taken and mixed 1:2 with TUD buffer (7 M Urea, $2 \mathrm{M}$ Thiourea, 2\% (w/v) DTT). For gel filtration, a Superose $610 / 300$ column ( $24 \mathrm{~mL}$ bedsize) (GE Healthcare) coupled to an Äkta U-900 FPLC system (GE Healthcare) was applied. Running buffer consisted of $7 \mathrm{M}$ urea, $2 \mathrm{M}$ thiourea, $1 \mathrm{mM}$ DTT. $500 \mu \mathrm{L}$ of sample was injected and fractions were collected as $500 \mu \mathrm{L}$ aliquots using a coupled Frac-900 sample fractionator (GE Healthcare).

\subsection{Dot Blot}

From each collected gel filtration fraction $1 \mu \mathrm{L}$ was spotted on a nitrocellulose membrane (Pall, Port Washington, NY, USA). The membrane was incubated with a directly HRP-conjugated monoclonal anti-CA125 antibody (M11-like, HyTest) at a 1:10.000 dilution and ECL detection (Sigma-Aldrich) was applied. 


\subsection{D Electrophoresis}

Proteins were precipitated using ReadyPrep 2D clean up kit (Bio-Rad) according to manufacturer's protocol. Proteins were resuspended in 1\% (w/v) SDS. Prior to 1D electrophoresis, $2 \%(w / v)$ DTT was added. HiMark prestained (Life Technologies) was used as a molecular mass standard. One-dimensional electrophoresis was carried out on $T=3-8 \%$ Tris-acetate gels (Life Technologies) according to manufacturers protocol. Proteins were stained with Coomassie brilliant blue overnight.

\subsection{Tryptic Digest}

Bands of interest were excised from the gel manually, destained, reduced with DTT and alkylated with Iodoacetamide (IAA). Digestion was carried out overnight at $37{ }^{\circ} \mathrm{C}$ with $100 \mathrm{ng}$ of sequencing grade modified trypsin (Promega).

\subsection{Filter Aided Digest}

Protein concentration of fractions 13-17 derived from size exclusion chromatography were estimated using an EZQ assay (Life Technologies). Filter aided digest was carried out as described in [27]. Deviating from the protocol, $8 \mathrm{M}$ Urea was substituted by $6 \mathrm{M}$ guanidine-hydrochloride, $0.1 \mathrm{M}$ Tris-HCl, $5 \mathrm{mM}$ EDTA, pH 8.0, Lys-C by trypsin (Promega, Madison, WI, USA) and $0.5 \mathrm{M} \mathrm{NaCl}$ by $40 \mathrm{mM}$ ammonium bicarbonate. Sample was evaporated in an Alpha 2-4 LD plus vacuum freeze-drier (Martin Christ Gefriertrocknungsanlagen $\mathrm{GmbH}$, Osterode am Harz, Germany) and the peptides were resuspended in FA2 (2\% acetonitrile, $0.1 \%$ formic acid and $97.9 \%$ water).

\subsection{Mass Spectrometry (HCT Ultra 3D ION Trap)}

Mass spectrometry was carried out on an 1100 series HPLC system (Agilent Technologies, Inc., Santa Clara, CA, USA) coupled to a HCT Ultra 3D-Ion-Trap mass spectrometer (Bruker Daltonik $\mathrm{GmbH}$, Bremen, Germany) as described previously [28].

\subsection{Mass Spectrometry (AmaZon 3D ION Trap)}

Mass spectrometry was performed using an online Prominance Nano HPLC system (Shimadzu, Nakagyo-ku, Kyoto, Japan) and amaZon 3D-Ion trap mass spectrometer (Bruker). The LC system was interfaced to the MS using an ESI nano Sprayer (Bruker). $5 \mu \mathrm{L}$ of sample was loaded on the $\mu$-Precolumn (Thermo Scientific) (Acclaim PepMap100 C18, $5 \mu \mathrm{m}, 100 \AA$, $300 \mu \mathrm{m}$ inner diameter. $\times 5 \mathrm{~mm})$ at a flow rate of $5 \mu \mathrm{L} / \mathrm{min}$ in Mobile Phase $\mathrm{A}\left(0.1 \%\right.$ formic acid (FA) in $\left.\mathrm{H}_{2} \mathrm{O}\right)$ and resolved on a $75 \mu \mathrm{m}$ inner diameter $\times 15 \mathrm{~cm}$, Acclaim PepMap100, C18, $3 \mu \mathrm{m}, 100 \AA$ (Thermo Scientific) using a 0-67\% gradient of Mobile Phase B $(0.1 \% \mathrm{FA}$ in $90 \% \mathrm{w} / \mathrm{v}$ acetonitrile $(\mathrm{ACN}))$ over $30 \mathrm{~min}$ at $400 \mathrm{~nL} / \mathrm{min}(0-60 \% \mathrm{ACN}$ over $30 \mathrm{~min})$. Ionizable species $(300<\mathrm{m} / \mathrm{z}<1500)$ were trapped and the three most intense ions eluting at the time were fragmented by collision-induced dissociation. Active exclusion was used to exclude a precursor ion for $0.2 \mathrm{~min}$ following the acquisition of two spectra. 


\subsection{Mass Spectrometry (LTQ Orbitrap XL)}

Peptides were separated on a HPLC system (Thermo Scientific) using a separation column (Thermo Scientific) (Acclaim PepMap RSLC, C18, pore size $100 \AA$, particle size $2 \mu \mathrm{m}, 75 \mu \mathrm{m}$ inner diameter (ID) $\times 15 \mathrm{~cm}$ length) and a trapping column (Thermo Scientific) (Acclaim PepMap100, C18, pore size $100 \AA$, particle size $3 \mu \mathrm{m}, 75 \mu \mathrm{m}$ ID $\oplus \times 2 \mathrm{~cm}$ length). HPLC system was coupled to a LTQ Orbitrap XL mass spectrometer (Thermo Scientific), using the following buffer system: (A) 2\% ACN, 0.1\% FA in water; (B) $80 \% \mathrm{ACN}, 0.1 \% \mathrm{FA}$ in water. For in-line desalting and concentration, $2 \mu \mathrm{L}$ of digest was loaded onto the trap column and then washed for $5 \mathrm{~min}$ with $100 \% \mathrm{~A}$ at $5 \mu \mathrm{L} / \mathrm{min}$ flow rate. Peptides were eluted at $300 \mathrm{~nL} / \mathrm{min}$ flow rate with the following $120 \mathrm{~min}$ gradient: $4 \% \mathrm{~B}$ for $10 \mathrm{~min}$, gradient to $55 \%$ B over $80 \mathrm{~min}$, gradient to $90 \% \mathrm{~B}$ in $30 \mathrm{~s}, 90 \% \mathrm{~B}$ for $9.5 \mathrm{~min}$, gradient from $90 \%$ to $4 \% \mathrm{~B}$ in $30 \mathrm{~s}$, $4 \%$ B for $19.5 \mathrm{~min}$. Full scan mass spectra were acquired in the Orbitrap over $\mathrm{m} / \mathrm{z} 300-2000$. The ten most intense ions at a threshold above 1000 were selected for collision-induced fragmentation in the linear ion trap at normalized collision energy of 35\% after accumulation to a target value of 1000 . Dynamic exclusion was enabled with a repeat count of 1 and an exclusion mass width by mass 1.50 below and above the precursor ion $\mathrm{m} / \mathrm{z}$. The same precursor was excluded for $30 \mathrm{~s}$.

\subsection{Data Analysis (HCT Ultra 3D ION Trap and AmaZon 3D ION Trap)}

MS and MS/MS spectra were subjected to peak detection and de-convolution using DataAnalysis (Version 3.4; Bruker: Bremen, Germany, 2006). Compound lists were exported into BioTools (Version 3.1; Bruker: Bremen, Germany, 2007) then submitted to MASCOT (Version 2.2; Matrix Science, Inc.: Boston, MA, USA, 2007). Peak list were searched against the Swiss Prot 2011_01 database, mammalian taxonomy. Carbamidomethylation of cysteine was set as a fixed modification and oxidation of methionine was set as variable modification. Peptide tolerance was allowed to be $\pm 0.3 \mathrm{Da}$, fragment mass tolerance was allowed to be $\pm 0.4 \mathrm{Da}$, with a maximum of 2 missed cleavages.

\subsection{Data Analysis (LTQ Orbitrap XL)}

Raw data files were subjected to the Proteome Discoverer software (Thermo Scientific) to set up the workflow, files were then submitted to MASCOT (Version 2.2; Matrix Science, Inc.: Boston, MA, USA, 2007) by the Proteome Discoverer Daemon (Thermo Scientific). Peak lists in the range from $350 \mathrm{~m} / \mathrm{z}$ to $5000 \mathrm{~m} / \mathrm{z}$ were searched against the Swiss Prot 2011_08 database, mammalian taxonomy and the option to match against a MASCOT derived decoy database was set. Carbamidomethylation of cysteine was set as a fixed modification and oxidation of methionine was set as variable modification. Peptide tolerance was allowed to be $\pm 10 \mathrm{ppm}$; fragment mass tolerance was allowed to be $\pm 0.6 \mathrm{Da}$, with a maximum of 2 missed cleavages.

\subsection{First Generation Elisa}

ELISA kit for the detection of CA125 levels in human serum was obtained from Abnova (Taipei City, Taiwan) (Lot No. RN-41425) and used according to the manufacturer's protocol. P95 serum samples were diluted 1:10 and 1:20 in $0.9 \%(\mathrm{w} / \mathrm{v}) \mathrm{NaCl}$ in water, fibronectin (MP Biomedicals, Solon, $\mathrm{OH}$, USA) was resuspended in $0.9 \%(\mathrm{w} / \mathrm{v}) \mathrm{NaCl}$ in water and $80 \mu \mathrm{g}$ fibronectin were spiked into P95 
serum to obtain a dilution of 1:20. Unspiked 1:10 and 1:20 diluted P95 serum was measured in triplicates, spiked 1:20 diluted P95 serum was measured in sextuplicate for ELISA. The plate was scanned in a Biotrack II plate reader (GE Healthcare) at $450 \mathrm{~nm}$. Standard curve was obtained by plotting absorbance of the manufacturer's standards against their CA125 concentration (Figure 3(A,B)). To test for statistically significant differences in CA125 readings, the data obtained for the 1:20 diluted P95 serum was compared to the spiked 1:20 diluted P95 serum by a Mann-Whitney $U$ test [29] (Figure 3(B,C)).

\subsection{Second Generation Elisa}

Apo-serotransferrin (Merck) and holo-serotransferrin (Merck) were resuspended in $\mathrm{H}_{2} \mathrm{O}$ to a concentration of $100 \mathrm{mg} / \mathrm{mL}$ and $100 \mu \mathrm{L}$ were mixed with $500 \mu \mathrm{L}$ serum of P607. As a control, $100 \mu \mathrm{L} \mathrm{H} \mathrm{H}_{2} \mathrm{O}$ was added to $500 \mu \mathrm{L}$ of P607 serum. All three samples were given to the IMVS to measure the CA125 concentration. The samples were measured in quintuplicate and tested for statistically significant differences by a Mann-Whitney U test [29] (Figure 3D).

\section{Conclusions}

The results of this study suggest that experimental findings involving the identification and quantification of CA125 should be viewed with caution when they are based on antibody probing alone and have not been verified by detailed mass spectrometric analysis. It is likely that proteins giving false-positive signals are the reason for the conflicting data on the molecular structure of CA125. Furthermore our results show that care should be taken for diagnostics, as the clinical determination of CA125 levels relies exclusively on ELISAs. As a way to overcome potential interferences of CA125 ELISAs with other proteins, an MRM mass spectrometric approach could be applied [30]. Our mass spectrometry results suggest that an assay based on SEC followed by an MRM quantification of several proteotypic peptides of CA125, using triple-quadrupole mass spectrometry instruments and isotopic labeled peptides as internal standards, is achievable.

\section{Acknowledgments}

This study was supported by the Ovarian Cancer Research Foundation, Australia. The authors wish to thank James Eddes and Yin Ying Ho for their assistance in acquiring the mass spectrometric data.

\section{Conflict of Interest}

The authors declare no conflict of interest.

\section{References}

1. Bast, R.C., Jr. Status of tumor markers in ovarian cancer screening. J. Clin. Oncol. 2003, 21, 200S-205S.

2. Bast, R.C., Jr.; Feeney, M.; Lazarus, H.; Nadler, L.M.; Colvin, R.B.; Knapp, R.C. Reactivity of a monoclonal antibody with human ovarian carcinoma. J. Clin. Invest. 1981, 68, 1331-1337. 
3. Davelaar, E.M.; van Kamp, G.J.; Verstraeten, R.A.; Kenemans, P. Comparison of seven immunoassays for the quantification of CA 125 antigen in serum. Clin. Chem. 1998, 44, 1417-1422.

4. Bast, R.C., Jr.; Klug, T.L.; St John, E.; Jenison, E.; Niloff, J.M.; Lazarus, H.; Berkowitz, R.S.; Leavitt, T.; Griffiths, C.T.; Parker, L.; et al. A radioimmunoassay using a monoclonal antibody to monitor the course of epithelial ovarian cancer. N. Engl. J. Med. 1983, 309, 883-887.

5. Bast, R.C., Jr.; Xu, F.J.; Yu, Y.H.; Barnhill, S.; Zhang, Z.; Mills, G.B. CA 125: The past and the future. Int. J. Biol. Markers 1998, 13, 179-187.

6. Seki, K.; Kikuchi, Y.; Uesato, T.; Kato, K. Increased serum CA 125 levels during the first trimester of pregnancy. Acta Obstet. Gynecol. Scand. 1986, 65, 583-585.

7. Miralles, C.; Orea, M.; Espana, P.; Provencio, M.; Sanchez, A.; Cantos, B.; Cubedo, R.; Carcereny, E.; Bonilla, F.; Gea, T. Cancer antigen 125 associated with multiple benign and malignant pathologies. Ann. Surg. Oncol. 2003, 10, 150-154.

8. Davis, H.M.; Zurawski, V.R., Jr.; Bast, R.C., Jr.; Klug, T.L. Characterization of the CA 125 antigen associated with human epithelial ovarian carcinomas. Cancer Res. 1986, 46, 6143-6148.

9. De los Frailes, M.T.; Stark, S.; Jaeger, W.; Hoerauf, A.; Wildt, L. Purification and characterization of the CA 125 tumor-associated antigen from human ascites. Tumour. Biol. 1993, $14,18-29$.

10. Kobayashi, H.; Ida, W.; Terao, T.; Kawashima, Y. Molecular characteristics of the CA 125 antigen produced by human endometrial epithelial cells: Comparison between eutopic and heterotopic epithelial cells. Am. J. Obstet. Gynecol. 1993, 169, 725-730.

11. Matsuoka, Y.; Nakashima, T.; Endo, K.; Yoshida, T.; Kunimatsu, M.; Sakahara, H.; Koizumi, M.; Nakagawa, T.; Yamaguchi, N.; Torizuka, K. Recognition of ovarian cancer antigen CA125 by murine monoclonal antibody produced by immunization of lung cancer cells. Cancer Res. 1987, 47, 6335-6340.

12. O'Brien, T.J.; Tanimoto, H.; Konishi, I.; Gee, M. More than 15 years of CA 125: What is known about the antigen, its structure and its function. Int. J. Biol. Markers 1998, 13, 188-195.

13. Yin, B.W.; Lloyd, K.O. Molecular cloning of the CA125 ovarian cancer antigen: Identification as a new mucin, MUC16. J. Biol. Chem. 2001, 276, 27371-27375.

14. Nustad, K.; Lebedin, Y.; Lloyd, K.O.; Shigemasa, K.; de Bruijn, H.W.; Jansson, B.; Nilsson, O.; Olsen, K.H.; O'Brien, T.J. Epitopes on CA 125 from cervical mucus and ascites fluid and characterization of six new antibodies. Third report from the ISOBM TD-1 workshop. Tumour Biol. 2002, 23, 303-314.

15. Shojaeian, S.; Allameh, A.; Zarnani, A.H.; Chamankhah, M.; Ghods, R.; Bayat, A.A.; Jeddi-Tehrani, M. Production and characterization of monoclonal antibodies against the extracellular domain of CA 125. Immunol. Invest. 2010, 39, 114-131.

16. Kui Wong, N.; Easton, R.L.; Panico, M.; Sutton-Smith, M.; Morrison, J.C.; Lattanzio, F.A.; Morris, H.R.; Clark, G.F.; Dell, A.; Patankar, M.S. Characterization of the oligosaccharides associated with the human ovarian tumor marker CA125. J. Biol. Chem. 2003, 278, 28619-28634. 
17. Elschenbroich, S.; Ignatchenko, V.; Clarke, B.; Kalloger, S.E.; Boutros, P.C.; Gramolini, A.O.; Shaw, P.; Jurisica, I.; Kislinger, T. In-depth proteomics of ovarian cancer ascites: Combining shotgun proteomics and selected reaction monitoring mass spectrometry. J. Proteome Res. 2011, 10, 2286-2299.

18. Milutinovic, B.; Jankovic, M. Analysis of the protein and glycan partsof CA125 Antigen from human amniotic fluid. Arch. Biol. Sci. Belgrade 2007, 59, 97-103.

19. Andersch-Bjorkman, Y.; Thomsson, K.A.; Holmen Larsson, J.M.; Ekerhovd, E.; Hansson, G.C. Large scale identification of proteins, mucins, and their $O$-glycosylation in the endocervical mucus during the menstrual cycle. Mol. Cell. Proteomics 2007, 6, 708-716.

20. Bouanene, H.; Miled, A. Conflicting views on the molecular structure of the cancer antigen CA125/MUC16. Dis. Markers 2010, 28, 385-394.

21. Klar, E.; Heene, D.L. Fibronectin. Klin. Wochenschr. 1984, 62, 963-974.

22. Kumar, V.; Hagler H.K. Interactive Case Study Companion to Robbins Pathologic Basis of Disease, 6th ed.; Saunders/Elsevier: Philadelphia, PA, USA, 1999.

23. Makarov, A.; Denisov, E.; Lange, O.; Horning, S. Dynamic range of mass accuracy in LTQ Orbitrap hybrid mass spectrometer. J. Am. Soc. Mass. Spectrom. 2006, 17, 977-982.

24. Milutinovic, B.; Mitic, N.; Jankovic, M. Identification of pregnancy-associated CA125-reactive protein as a carbohydrate-binding immunoglobulin G. Arch. Biochem. Biophys. 2010, 499, 69-76.

25. Hanisch, F.G.; Uhlenbruck, G.; Dienst, C.; Stottrop, M.; Hippauf, E. Ca 125 and Ca 19-9: Two cancer-associated sialylsaccharide antigens on a mucus glycoprotein from human milk. Eur. J. Biochem. 1985, 149, 323-330.

26. Gorg, A.; Drews, O.; Luck, C.; Weiland, F.; Weiss, W. 2-DE with IPGs. Electrophoresis 2009, 30, S122-S132.

27. Wisniewski, J.R.; Zougman, A.; Nagaraj, N.; Mann, M. Universal sample preparation method for proteome analysis. Nat. Methods 2009, 6, 359-362.

28. Penno, M.A.; Bacic, A.; Colegate, S.M.; Hoffmann, P.; Michalski, W.P. Identifying candidate serum biomarkers of exposure to tunicamycins in rats using two-dimensional electrophoresis. J. Proteome Res. 2009, 8, 2812-2826.

29. Avery, L. Mann-Whitney U Test. 2007. Available online: http://elegans.som.vcu.edu/ leon/stats/ utest.html (accessed on 3 May 2012).

30. Hoofnagle, A.N.; Becker, J.O.; Wener, M.H.; Heinecke, J.W. Quantification of thyroglobulin, a low-abundance serum protein, by immunoaffinity peptide enrichment and tandem mass spectrometry. Clin. Chem. 2008, 54, 1796-1804.

(C) 2012 by the authors; licensee MDPI, Basel, Switzerland. This article is an open access article distributed under the terms and conditions of the Creative Commons Attribution license (http://creativecommons.org/licenses/by/3.0/) 